12 POSITIVE AIRWAY PRESSURE ADHERENCE AND REMOTE VERSUS FACE-TO-FACE EDUCATION FOR SLEEP APNOEA DURING THE NOVEL CORONAVIRUS (COVID-19) PANDEMIC

${ }^{1} J o h a n$ Meurling*, 'Adam Birdseye, ${ }^{1}$ Rohan Gell, ${ }^{1}$ Eliza Sany, ${ }^{1}$ Richard Brown, ${ }^{1}$ Sean Higgins, ${ }^{1}$ Rexford Muza, ${ }^{1,2}$ David O' Regan, ${ }^{1,3,4}$ Guy Leschziner, ${ }^{1,5}$ Joerg Steier, ${ }^{1,3}$ Ivana Rosenzweig, 1,5Panagis Drakatos. 'Sleep Disorders Centre, Guys and St. Thomas' NHS Foundation Trust, London, UK; ${ }^{2}$ School of Medicine, King's College London, London, UK; ${ }^{3}$ Plasticity Centre, Department of Neuroimaging, Institute of Psychiatry, Psychology and Neuroscience (IOPPN), King's College London, London, UK; ${ }^{4}$ Department of Neurology, Guy's and St Thomas' NHS Foundation Trust, London, UK; ${ }^{5}$ Faculty of Life Sciences and Medicine, King's College London, London, UK

\title{
10.1136/bmjresp-2021-bssconf.10
}

Introduction We aim to quantify adherence of positive airway pressure (PAP) initiated during the COVID-19 pandemic and compare the effects of remote versus face-to-face (FTF) education in patients with obstructive sleep apnoea (OSA).

Methods Prospective cohort study in a tertiary National Health Service (NHS) hospital sleep disorders centre in London, United Kingdom, involving 141 patients aged $>18$ years with newly diagnosed OSA initiating PAP during the first national coronavirus (COVID-19) lockdown in 2020. We compare 70 patients educated on PAP remotely to 71 patients receiving standard FTF education. We measured adherence over a continuous 30-day period within the first three months of PAP usage, including average nightly usage, usage per nights used, percentage of nights used, and percentage of nights used for $>4$ hours. 
Results Of 141 patients, there was a two-thirds male predominance, and half of the patients (56\%) above 45 years of age and sleepy at baseline (Epworth Sleepiness Score $>10,48.9 \%$ ). 114 patients (81\%) were diagnosed with moderate or severe OSA. 54 patients (38.3\%) achieved good adherence $(\geq 70 \%$ of nights with $\geq 4$ hours usage), with an average of 4.7 hours of PAP usage per night used. Patients receiving FTF PAP education had a comparable level of good adherence (38.03\% versus 38.57\%, p=0.915), and hours per nights used (4.76 versus $4.61 \mathrm{~h} / \mathrm{night}$, $\mathrm{p}=0.711)$ to remotely educated patients. More severe OSA, lower mask leak, and a nasal mask were associated with achieving good PAP adherence.

Discussion PAP adherence of newly diagnosed individuals with OSA during the COVID-19 pandemic was modest at $38.30 \%$, and not significantly affected by remote PAP education delivery, but rather the effects of the COVID-19 pandemic.

\section{THE EFFECT OF CONTINUOUS POSITIVE AIRWAY PRESSURE (CPAP) ON KEY PLASMA METABOLITES IDENTIFYING OBSTRUCTIVE SLEEP APNOEA HYPOPNOEA SYNDROME (OSAHS)}

${ }^{1}$ Scott O'Rourke*, ${ }^{2}$ Sarah Thomas, ${ }^{1}$ Catrin Lewis, ${ }^{1}$ Sarah Bowen, ${ }^{2}$ Luis Mur, ${ }^{1}$ Keir Lewis. ${ }^{1}$ Prince Phillip Hospital, Llanelli, UK; ${ }^{2}$ Aberystwyth University, Aberystwyth, UK

10.1136/bmjresp-2021-bssconf.11

We recently applied untargeted metabolomic profiling on the plasma obtained from consecutive attenders referred for conventional Level 3 home-sleep studies with excessive daytime somnolence, comparing 17 OSAHS patients $(\mathrm{AHI} \geq 15$, Epworth Score $13.5 \pm 4.5$ ) with 16 age, gender, and BMI matched sleepy subjects (sleepy snorers (SS)) with negative home polysomnography tests $(\mathrm{AHI}<15$, Epworth Score 12.1 $\pm 7.0)^{1}$

We reported 6 biologically plausible plasma metabolites that can differentiate OSAHS from SS of similar phenotype with an AUC of 0.982 (95\% CI: 0.9-1.0) (figure 1), with these key metabolites being essential lipids involved in protein synthesis and the formation of antioxidative, antiglycating, and free radical scavenging dipeptides. We now report early changes in these biomarkers following CPAP in those with OSAHS.

11 OSAHS patients with $\mathrm{AHI} \geq 15$ (63.6\% male, Age 54.4 \pm 6.9 , BMI 34.2 \pm 4.0 , AHI 47.6 \pm 25.6 , Epworth 13.7 \pm 4.8 ) were commenced on standard auto-adjustment CPAP devices (Phillips DreamStation set at 4 to $18 \mathrm{~cm} \mathrm{H2O}$ ). Mean use of CPAP was $6.6 \pm 1.4$ hours and average residual AHI was 6.9 \pm 6.0 . Plasma was sampled pre and post treatment (42-70 days treatment), and metabolomically assessed using the $Q$ Exactive Hybrid Quadrupole-Orbitrap mass spectrometry platform. 16 sleepy snorers with AHI $<15$ (75.0\% male, Age 46.1

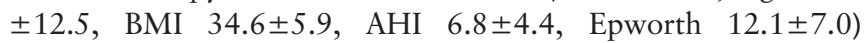
were sampled at baseline only.

Our previously reported biomarkers associated with processes such as oxidative stress, inflammation, and dysregulation of energy homeostasis improve with short-term treatment with CPAP towards the level of sleepy snorers of similar age, phenotype, and no OSAHS (figure 2). We feel these metabolites have significant potential in the future care pathways of

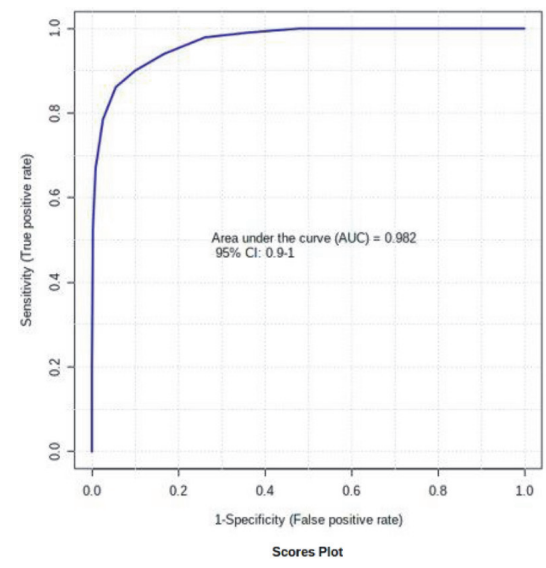

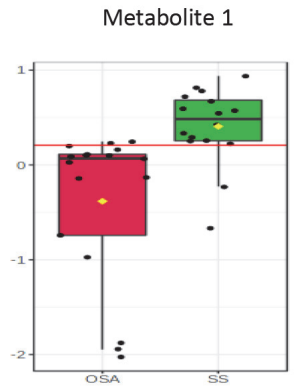
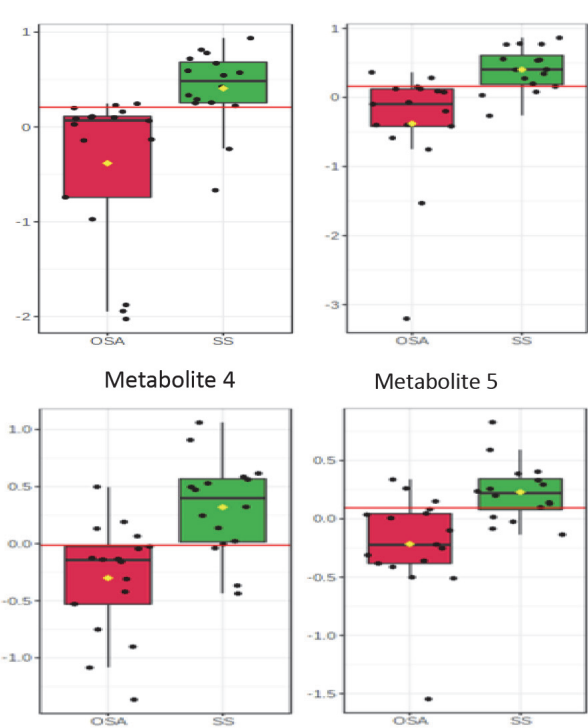

Metabolite 5
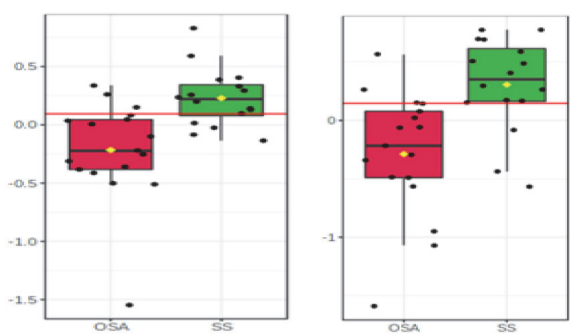

Abstract 13 Figure 1 Metabolomic discrimination between the plasma of OSAHS and SS patients 